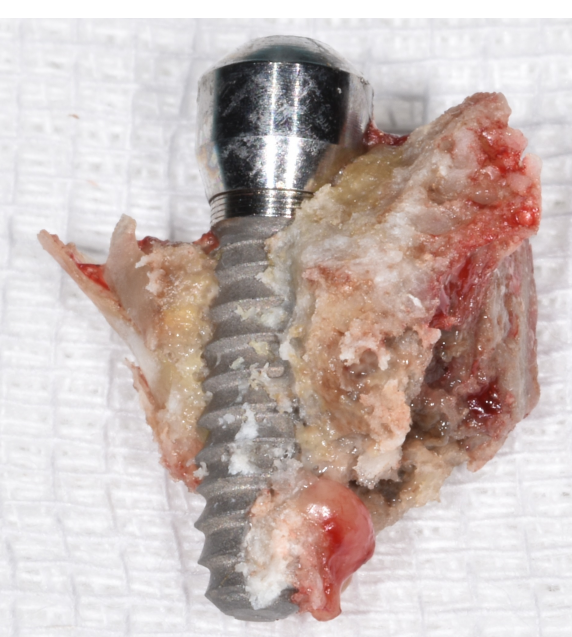

\title{
MRONJ ASSOCIATED WITH DENTAL INFECTION AND SECONDARY INVOLVEMENT OF THE NEARBY IMPLANT: A CASE REPORT
}

\author{
Stefania Nespoli ${ }^{1}$, Elisabetta Audino ${ }^{1}$, luca visconti, Francesco Daleffe ${ }^{2}$, Andrea Castellani, Stefano Negrini ${ }^{2}$, Stefano \\ Salgarello ${ }^{1}$, Magda MENSI ${ }^{1}$ \\ 1 University of Brescia \\ 2 ASST Spedali Civili
}

Funding: The author(s) received no specific funding for this work.

Potential competing interests: The author(s) declared that no potential competing interests exist.

\section{Abstract}

Background: Medication related osteonecrosis of the jaw (MRONJ) represents a potential adverse effect related to the treatment with antiresorptive (Bisphosphonates, Denosumab) and antiangiogenic (Tyrosine kinase inhibitors, Vascular endothelial growth factor inhibitors) drugs. This condition could occur in patients that take these agents for the treatment of skeletal and oncological diseases but have no history of radiation therapy to the jaws. MRONJ is characterized by exposed bone or bone that can be probed through an intraoral or extraoral fistula in maxillofacial region that has persisted for longer than 8 weeks ${ }^{[1]}$. The risk to develop osteonecrosis increases with local factors, especially dental infection and dentoalveolar surgery such as tooth extraction.

Case presentation: This report describes a case of bisphosphonate related to osteonecrosis of the jaw associated with tooth infection and subsequent involvement of the nearby implant.

Conclusion: The aim of this case is to describe the decision-making and clinical management of MRONJ carried out in ambulatory way and underline the importance of anamnesis and early diagnosis. 


\section{INTRODUCTION}

Bisphosphonates (BPs) represent a class of drugs used in treatment of diseases involving the skeletal system. The advantage is the effectiveness in reducing incidence of bone metastases in cancer and hematological patients (i.e. solid tumors and multiple myeloma), Paget's disease and osteoporosis ${ }^{[2]}$. BPs nitrogen containing drugs (such as Alendronate, Risedronate, Pamidronate, Ibandronate Zoledronate), parenterally and orally assumed, accumulate on hydroxyapatite crystals in bone resorption sites. Then BPs are endocytosed by osteoclasts inhibiting the mevalonate enzymatic pathway that leads in a significant reduction of bone resorption and consequent increase of bone density ${ }^{[3]}$. Due to bone attachment, depending on chemical and biological factors as well as type, quantity and intake system, BPs accumulate in the body over time ${ }^{[4]}$. While taking these drugs has a benefit for the patient's underlying pathology, the main adverse effect is the development of $\mathrm{ONJ}$, i.e. a reaction characterized by exposed bone or bone that can be probed through an intraoral or extraoral fistula and persists for more than 8 weeks in patients with no history of radiotherapy of the jaw or evident metastatic disease of the jaw ${ }^{[1]}$. Risk factors in developing ONJ are represented by patient's pathology, type of BPs, way and timing of administration of the antiresorptive drug and local factors. Considering the latter, oral surgery (tooth extraction) is the most common trigger factor in developing $\mathrm{ONJ}$, followed by peri-implant and periodontal infections ${ }^{[5]}$. Therefore, prevention through dental screening before starting any ONJ related drug and regular checks with oral hygiene sessions, are fundamental measures to reduce the risk of developing osteonecrosis of the jaws ${ }^{[6]}$.

\section{MATERIALS and METHODS}

In October 2019, an 84-year-old Caucasian woman accessed to the Dental Emergency Room inside Dental School of University of Brescia. The patient lamented acute pain, right jaw swelling and suppurative drainage. Intraoral examination reported vestibular swelling, multiple fistulas, mobility of the canine (13), and two non-prosthetically loaded dental implants (site 14 and site 15). Anamnesis reported allergy to Penicillins, assumption of Cardioaspirin and Bisoprolol due to previous heart attack, Olmesartan for hypertension and Statins for hypercholesterolemia. Since the patient at first did not report the assumption of antiresorptive drugs, a diagnosis of periodontal abscess (radiographically presence of periradicular lesion) was made, antibiotic therapy with Ciprofloxacin was prescribed and sent to her dentist. In November 2019, the patient returned to the Dental Emergency Room showing results of canine extraction, but again complaining of pain, swelling, nasal drainage, without any improvement in the symptoms referred to the previous access. It had been necessary a more specific pharmacological and medical history to reveal that the patient had been treated with oral alendronate (70 mg / week) for more than ten years for osteoporosis. Intraoral evaluation presented: vestibular swelling, soft tissue healing of the post-extraction socket, leakage of exudate with multiple fistulas (in correspondence of dental elements 13 and 14), but absence of bone exposure. Suspect of MRONJ (stage I) was made and a CBCT was prescribed. Then, evaluation of CBCT showed radiolucency at the level of the post-extraction socket (13) and around the dental implant (14). Combining imaging and clinical symptoms (pathological probing associated with suppuration and profuse bloody probing), the diagnosis of MRONJ, originating from the infection of 13 and extended to the first implant (site 14), was confirmed. Consequently, a surgical revision was planned and Moxifloxacin $400 \mathrm{mg}$ / day and rinses with chlorhexidine $0.2 \% 3$ days before the surgical intervention was prescripted. In January 2020, after professional oral hygiene and previous local 
anesthesia, was performed a sequestrectomy surgery that required a full thickness flap extended from the 13 to the 15 element (14 -15 implant elements) in order to clinically valuate the eventual overextension of the necrotic bone on the second implant. The ONJ extended from the post-extraction socket of the element 13 to the implant in site 14 , without involving the maxillary sinus (absence of oro-antral communication following a Valsalva maneuver) and the adjacent implant in site 15, which was therefore preserved. The debridement of the necrotic bone was performed and the implant in situ 14 was removed. Then collagen sponges (SuprasorbTM $C^{\circledR}$ ) were inserted into the surgical site and the flap was sutured to obtain a primary intention healing. A week after surgery, good healing of the post-surgical site was observed in absence of symptoms. The sutures were removed 14 days later, followed check-ups at 1-3 months, in accordance with the Covid-19 pandemic. In September 2020, barely 8 months after the first access, the treated site was perfectly healed, there were not swelling, fistulas or suppuration on probing. The implant at level 15, which was maintained because not involved by the necrotic process, was osseointegrated, healthy and with physiological probes. The patient was prosthetically rehabilitated by her dentist with a removable partial prosthesis with conometric support on implant in place 15 and resumed therapy with oral Alendronate. Finally, was recommended six-monthly checks and professional oral hygiene to the dentist and send to our department for follow up.

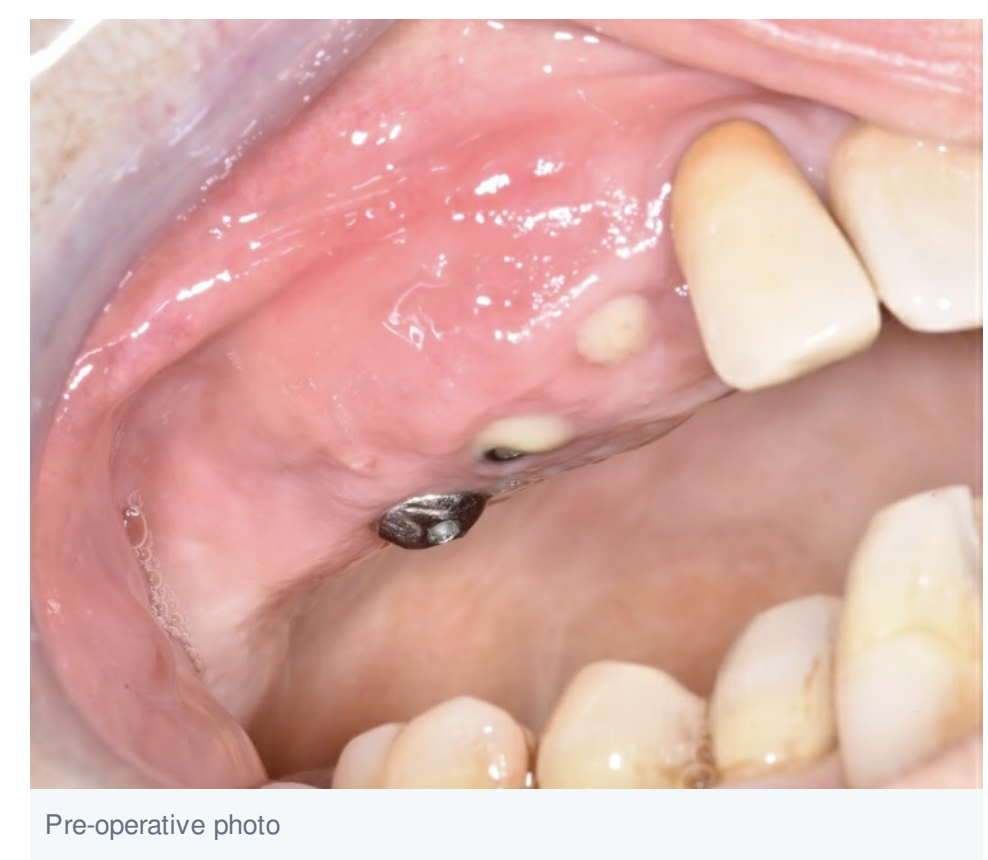




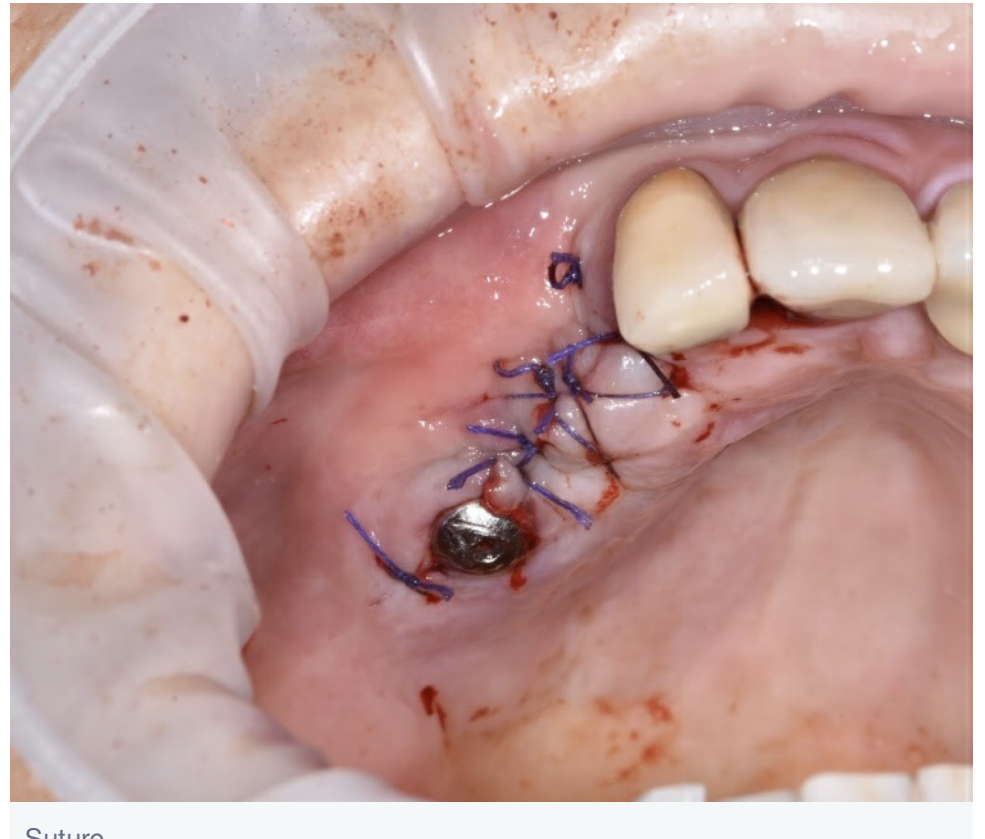

Suture

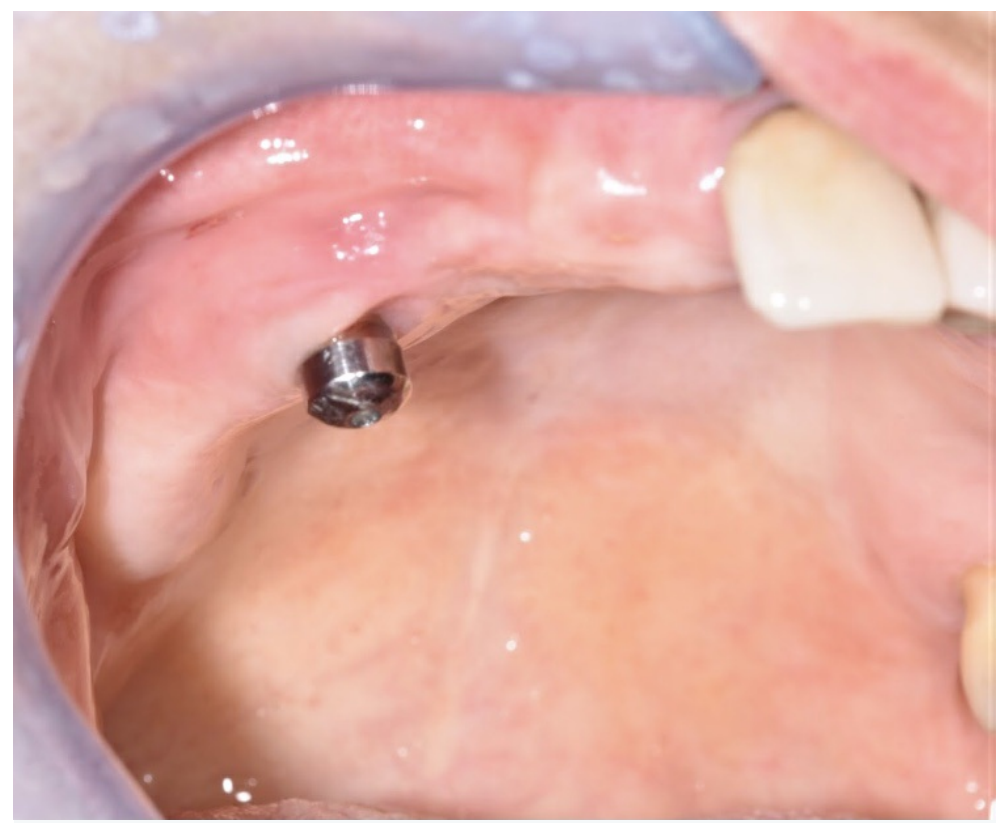

8 month follow-up

\section{DISCUSSION}

The case report describes an example of development and management of osteonecrosis of the jaw in a patient treated with BPs orally. In literature, prevalence of ONJ development in patients taking BPs orally for osteoporosis is lower compared to patients taking BPs intravenously for oncological diseases ${ }^{[7]}$. The difference could be related to reduced absorption of the drug from the oral intake rather than the parenteral intake. Despite this, as observed in this case, prolonged timing of drug intake represents an important risk factor for the development of ONJ as this is related to its dosetime intaking. In fact, is necessary at least 3 years of orally assumption of BPs to develop the adverse event ${ }^{[4]}$. In addition to the assessment of the risk related to systemic and local factors, to diagnose osteonecrosis is necessary starting from a diagnostic suspicion that it is subsequently ascertained by an imaging examination. In the case reported, despite the 
anamnesis carried out in the first session, the patient failed to reveal the assumption of the antiresorptive drug. Only afterwards, following a more accurate study of the pharmacological history, emerged the previous therapy with alendronate for more than 10 years. Therefore, the diagnostic suspicion of MRONJ was made and prescribing CBCT became necessary. The evaluation of $\mathrm{CBCT}$ is essential to obtain a diagnostic assessment, especially in cases where, like the one described, there is no evidence of bone exposure, but clinical signs characteristic of stage 0 and 1 of the disease remained (according to the AAOMS staging ${ }^{[1]}$ ): multiple mucous fistulas, swelling, pain, second degree mobility and radiographically peri-radicular radiolucency. In this case report, the local factor triggering ONJ could be referred to the periodontal disease of element 13 (with subsequent involvement of the implant in site 14) or conversely from a periimplantitis of element 14 . The treatment and management of MRONJ respected the protocols recommended in Italy by SICMF and SIPMO. In particular, as regards stage 1 (focal ONJ) as in this case, a combined medical-surgical therapy was performed under local anesthesia, that consisted of dentoalveolar curettage, sequestrectomy at the level of the implant involved, topical antiseptic therapy, perioperative systemic antibiotic therapy and BP suspension until biological healing of the tissues. The patient was then monitored during follow-up checks observing excellent tissue healing and was successfully prosthetically rehabilitated by the dentist. Now she is currently part of the MRONJ prevention and follow-up program. Although 18 years have passed since the first report of osteonecrosis related to the intake of BPs (Marx and Coll $2003^{[8]}$ ), the risks associated with this type of therapy are often still unknown to patients. This phenomenon may be attributable to the lack of effective and understandable communication between the healthcare professional and the patient regarding the benefit of the treatment and the possible side effects associated with it. The responsibility for the manifestation of the adverse advent (MRONJ) is equally shared between the prescribing doctor, dentist, treating doctor who manages comorbidities and patient ${ }^{[9]}$. Consequently, only appropriate patient education and healthcare professional knowledge on the subject leads to the correct management and prevention of the adverse event, focusing on importance of primary and secondary prevention.

\section{References}

1. a, b, c Noam Yarom, Charles L. Shapiro, Douglas E. Peterson, Catherine H. Van Poznak, et al. (2019). MedicationRelated Osteonecrosis of the Jaw: MASCC/ISOO/ASCO Clinical Practice Guideline. JCO, vol. 37 (25), 2270-2290. doi:10.1200/jco.19.01186.

2. 'Ourania Nicolatou-Galitis, Morten Schiødt, Rui Amaral Mendes, Carla Ripamonti, et al. (2019). Medication-related osteonecrosis of the jaw: definition and best practice for prevention, diagnosis, and treatment. Oral Surgery, Oral Medicine, Oral Pathology and Oral Radiology, vol. 127 (2), 117-135. doi:10.1016/j.00oo.2018.09.008.

3. 'Matthew R Allen. (2008). Skeletal accumulation of bisphosphonates: implications for osteoporosis treatment. Expert Opinion on Drug Metabolism \& Toxicology, vol. 4 (11), 1371-1378. doi:10.1517/17425255.4.11.1371.

4. a, bPPL Fung, G Bedogni, A Bedogni, A Petrie, et al. (2017). Time to onset of bisphosphonate-related osteonecrosis of the jaws: a multicentre retrospective cohort study. Oral Dis, vol. 23 (4), 477-483. doi:10.1111/odi.12632. 
5. ' K McGowan, T McGowan, S Ivanovski. (2017). Risk factors for medication-related osteonecrosis of the jaws: A systematic review. Oral Dis, vol. 24 (4), 527-536. doi:10.1111/odi.12708.

6. 'Olga Di Fede, Vera Panzarella, Rodolfo Mauceri, Vittorio Fusco, et al. (2018). The Dental Management of Patients at Risk of Medication-Related Osteonecrosis of the Jaw: New Paradigm of Primary Prevention. BioMed Research International, vol. 2018 , 1-10. doi:10.1155/2018/2684924.

7. 'Salvatore L. Ruggiero, Thomas B. Dodson, John Fantasia, Reginald Goodday, et al. (2014). American Association of Oral and Maxillofacial Surgeons Position Paper on Medication-Related Osteonecrosis of the Jaw-2014 Update. Journal of Oral and Maxillofacial Surgery, vol. 72 (10), 1938-1956. doi:10.1016/j.joms.2014.04.031.

8. 'Robert E Marx. (2003). Pamidronate (Aredia) and zoledronate (Zometa) induced avascular necrosis of the jaws: a growing epidemic. Journal of Oral and Maxillofacial Surgery, vol. 61 (9), 1115-1117. doi:10.1016/s02782391(03)00720-1.

9. 'L Lo Russo, L Lo Muzio, C Buccelli, P Lorenzo. (2012). Bisphosphonates-related osteonecrosis of the jaws: medicolegal issues. Oral Dis, vol. 19 (4), 425-426. doi:10.1111/odi.12049. 